\title{
Capillary Electrophoresis Fourier Transform Ion Cyclotron Resonance Mass Spectrometry with Sustained Off-Resonance Irradiation for the Characterization of Protein and Peptide Mixtures
}

\author{
Steven A. Hofstadler, Jon H. Wahl, Ray Bakhtiar*, Gordon A. Anderson, \\ James E. Bruce, and Richard D. Smith \\ Chemical Sciences Department, Pacific Northwest Laboratory, P.O. Box 999, MS P8-19, Richland, \\ Washington, USA
}

\begin{abstract}
A new approach to protein and peptide analysis that involves the coupling of on-line capillary electrophoresis-electrospray ionization Fourier transform ion cyclotron resonance mass spectrometry with a variation of sustained off-resonance irradiation is described. With this technique, multiple irradiation frequencies are broadcast simultaneously, which yields fragmentation of species at different mass-to-charge ratio values from the same waveform. In conjunction with capillary electrophoresis, this technique can provide sequence information from small amounts of proteins or peptides in complex mixtures. Initial results obtained from a mixture of gramicidin $S(1141 \mathrm{u}$ ), bee venom melittin (2845 $\mathrm{u}$ ), and equine apomyoglobin (16,951 u) are presented. (/ Am Soc Mass Spectrom 1994, 5, 894-899)
\end{abstract}

$\mathrm{T}$ The electrospray ionization (ESI) Fourier transform ion cyclotron resonance (FTICR) mass spectrometry combination pioneered by McLafferty and co-workers $[1,2]$ has demonstrated numerous advantages over conventional quadrupole mass spectrometric detection, including the ability to simultaneously realize ultra-high sensitivity [3], unparalleled resolving power and mass measurement accuracy [4-7], the capability for higher order tandem mass spectrometry methods (i.e., $\mathrm{MS}^{n}$, where $n \geq 2$ ) [1, $8-10]$, and the ability to nondestructively remeasure ion populations [11]. Recent advances in dissociation techniques such as surface-induced dissociation (SID) $[12,13]$ and sustained off-resonance irradiation (SORI) [14] are particularly appealing when used in conjunction with the nondestructive detection scheme inherent to FTICR. We have recently developed FTICR instrumentation that affords ultra-high resolution and allows rapid manipulation of pressures in the FTICR cell between those that are optimum for ion trapping and cooling ( $>10^{-5}$ torr) and those required for high resolution mass measurements ( $<10^{-8}$ torr) [4]. The ability to rapidly cycle between high pressure accumula-

Address reprint requests to Dr. Richard D. Smith, Chemical Methods and Separations Group, Chemical Sciences Department, Battelle, Pacific Northwest Laboratories, Battelle Boulevard, P8-19, P.O. Box g9g Richland, WA 99352

* Present address: Drug Metabolism Department, Merck Research Laboratories, P.O. Box 2000, Rahway, NJ 07065. tion-cooling events and low pressure detection events is an essential prerequisite for the implementation of high resolution mass analysis in tandem with a high resolution on-line separation.

In this communication we present initial results obtained from the coupling of on-line capillary electrophoresis-electrospray ionization Fourier transform ion cyclotron resonance (CE-ESI/FTICR) with SORI collision-activated dissociation [14] in which the SORI waveforms are composed of multiple frequencies. With this method multiple irradiation frequencies are broadcast simultaneously, which results in the dissociation of species at different mass-to-charge ratio values with the same waveform. In conjunction with capillary electrophoresis (CE), this scheme can provide complete or partial sequence information from very small amounts of proteins or peptides in complex mixtures.

\section{Experimental}

The instrumentation used in these studies has been described in considerable detail elsewhere [4]; thus, only a brief description is given here. The FTICR mass spectrometer utilized in this work is based on a 7-T superconducting magnet and is equipped with an external electrospray ionization source. Ions are transferred to the trapped ion cell by two sets of rf-only quadrupoles. Background pressure in the trapped ion cell is maintained at $10^{-9}-10^{-10}$ torr by a custom 
cryopumping assembly that consists of two sets of cryobaffels with radiation shields that are maintained at 77 and $14 \mathrm{~K}$, respectively, by closed cycle cryogenic compressors. The large surface area of the cryobaffels provides pumping speeds in excess of $10^{5} \mathrm{~L} / \mathrm{s}$, which permits rapid transitions between the high pressure $\left(10^{-4}\right.$ torr) ion accumulation event and the low pressure $\left(<10^{-9}\right.$ torr $)$ detection event. A typical CEESI/FTICR pulse sequence consists of five events: ion injection-accumulation, ion cooling-pump down, rf excitation, detection, and data transfer. The minimum time between ion accumulation and detection (i.e., pump-down time) for the described instrumentation is approximately $1 \mathrm{~s}$. However, present hardware and software limitations restrict data acquisition to approximately one spectrum every $5 \mathrm{~s}$. With the exception of the multiple frequency SORI waveforms, electronics and all aspects of pulse sequence timing were controlled by the Ionspec (Irvine, CA) Omega data station. Multiple frequency SORI waveforms were generated by custom software written at Pacific Northwest Laboratory (PNL) and created on a LeCroy (Chestnut Ridge, NY) arbitrary function generator.

The CE instrumentation used in these experiments is housed in a Plexiglas ${ }^{\circledast 2}$ box to isolate the high voltage components. In these studies, $50-\mu \mathrm{m}$-i.d. by 1-m-long fused-silica capillaries were utilized (Polymicro Technologies, Phoenix, AZ) in which the inner walls of the capillaries were chemically modified with 3-aminopropylsilane [3, 15, 16]. Approximately $1 \mathrm{~cm}$ of the polyimide coating was removed from the capillary terminus, and the exposed fused silica was etched with a $40 \%$ hydrofluoric acid solution (Aldrich, Milwaukee, WI) to taper the outlet of the analytical capillary. To the tapered $\mathrm{CE}$ outlet, a gold conductive coating (Epoxy Technology, Billerica, MA) was applied to allow electrospray directly from the $C E$ terminus $[17,18]$. The epoxy establishes electrical contact with the CE capillary terminus and assists in the electrospray process. This conductive gold coating also serves to establish electrical contact with the buffer solution at the CE capillary terminus. Advantages of this type of CE mass spectrometry electrospray tip include ease of use, spray stability, and reduced background contamination compared to a sheath-type ESI interface [17]. The electrospray is produced by using a $3.8-\mathrm{kV}$ gradient between the CE capillary terminus and the heated metal capillary inlet of the electrospray source. Coaxial to the fused-silica capillary, a sheath gas of sulfur hexafluoride $\left(\mathrm{SF}_{6}\right)$ is utilized to suppress corona discharge.

The buffer system used in all experiments is a 10-mM acetic acid solution ( $\mathrm{pH}$ 3.4). As a result of the aminopropylsilane surface modification and the acidic buffer system, the inner walls of the capillaries have a net positive charge that results in an electro-osmotic flow in the opposite direction compared to uncoated fused-silica capillaries. The electric field strength used for these studies was approximately $-290 \mathrm{~V} \mathrm{~cm}^{-1}$.
Analytes in this initial study were gramicidin S (1141 u), bee venom melittin ( $2845 \mathrm{u})$, and equine apomyoglobin (16,951 u) (Sigma, St. Louis, MO) in doubly distilled deionized water. A mixture of these components was electrokinetically injected at $\approx-38$ $\mathrm{V} \mathrm{cm}{ }^{-1}$ for $\approx 3 \mathrm{~s}$.

\section{Results and Discussion}

\section{Collision-Activated Dissociation}

Collision-activated dissociation (CAD) has been the most widely used technique for probing the structure of ions in the gas phase [19]. Ease of application to various instrument schemes, including FTICR, along with its experimental simplicity, account for the wide popularity of CAD. To date, CAD with FTICR has shown limited success in fragmenting high molecular weight ions partially because of the relatively low center-of-mass collision energy and because of dispersal of ions through the cell volume at high collision numbers (making subsequent detection problematic). Furthermore, the enormous number of vibrational modes $(3 N-6)$ requires that larger ions obtain internal energies that greatly exceed the dissociation threshold for fragmentation [20-22].

A number of novel alternatives for rendering the CAD of molecular ions in FTICR have been reported. Boering et al. [23] introduced an ion kinetic energy control scheme that continuously accelerates and decelerates ions on a millisecond time scale, which results in the alleviation of the radial ejection problem that plagues "on-resonance" FTICR-CAD. Lee et al. [24] developed a multiple excitation collisional activation approach in which ions are translationally excited to larger radii by application of a radiofrequency pulse that encompasses the specified mass range. Collisional cooling of ions facilitates their collapse to the center of the cell, which is followed by repetition of the multiple excitation pulse. This process is repeated for as many cycles as necessary to achieve ion fragmentation.

\section{Sustained Off-Resonance Irradiation}

Jacobson and co-workers [14] reported a method that involves sustained "off-resonance" irradiation (SORI) for sampling the lowest energy dissociation pathway. In this technique, ion excitation is achieved by application of an "off-resonance" electric field pulse, where the frequency is set some $500-2000 \mathrm{~Hz}$ away from the observed cyclotron frequency of the ion. The maximum translational energy (in the absence of collisions) of the ion is then described as

$$
E_{\text {tr(max) }}=\left\{\left(E_{\mathrm{rf}}\right)^{2} e^{2} /\left(2 m\left(\omega_{1}-\omega_{c}\right)^{2}\right)\right\} \sin ^{2}\left(\omega_{1}-\omega_{\mathrm{c}}\right) t / 2
$$

where $\omega_{1}$ (radians per second) is the excitation frequency, $\omega_{c}$ (radians per second) is the natural cy- 
clotron frequency of the ion, $E_{\text {trmax) }}$ is the laboratory frame translational energy of the ion, $E_{\mathrm{rf}}$ (volts per meter) is the electric field amplitude, $t$ is the duration of the electric field pulse (typically 500-2000 ms), and $e$ is the elemental electric charge. Consequently, by employing a long duration, "off-resonance" electric field pulse and an appropriate $\Delta \omega\left(\Delta \omega=\omega_{1}-\omega_{\mathrm{c}}\right)$, ions experience a series of acceleration-deceleration cycles throughout the duration of the electric field pulse. Thus, in the presence of a gas, ions can be slowly collisionally activated by sequential low energy collisions; generally with small dispersal from their initial location in the trap. The number of acceleration-deceleration cycles is given by

$$
n=t\left(f_{1}-f_{t}\right)
$$

where $f_{1}$ and $f_{c}$ are the frequencies (Hertz) of the applied electric field and the observed cyclotron frequency, respectively. High mass ions can be irradiated for long periods of time and undergo many collisions without being ejected from the cell. The average energy of the collisions is easily controlled by varying either the irradiation amplitude $\left(E_{\mathrm{rf}}\right)$ or the frequency difference between the effective cyclotron frequency and the irradiation frequency $(\Delta \omega)$. A pulse of $\mathrm{N}_{2}$ was admitted to the cell region as a target gas coinciding with the SORI event. Peak pressures were typically $10^{-5}$ torr.

A minor limitation of the SORI technique is the unintentional ejection of product ions, which may fall in close proximity to the irradiation frequency. The width of these "blind spots" varies with the irradiation duration and intensity. In a 7-T field, irradiating at $8.5 \mathrm{~V}$ for $0.5 \mathrm{~s}$ results in blind spots that are about 2 $\mathrm{kHz}$ wide. The mass-to-charge width of the blind spot is reduced at higher cyclotron frequencies (i.e., lower mass-to-charge ratio or higher magnetic field strength). For example, in a 7-T field a 2-kHz blind spot at $\mathrm{m} / \mathrm{z}$ 1000 corresponds to $18.6 \mathrm{~m} / z$ units, whereas the same 2-kHz blind spot at $m / z 500$ corresponds to only 4.6 $m / z$ units. At 12-T, $2 \mathrm{kHz}$ blind spots at $m / z 1000$ and 500 correspond to mass-to-charge ratio windows of only 10.9 and 2.8 , respectively. In cases where a product ion lands in a blind spot, the irradiation frequency can be moved to the other side of the parent ion (i.e., $-1 \times \Delta \omega$ ) thus effectively moving the blind spot. Thus, it is often desirable to perform two dissociation studies-one at $+\Delta \omega$ and one at $-\Delta \omega$ - to insure that no unique product ions are overlooked. Additionally, the width of the blind spot can be significantly reduced by applying an attenuated $E_{\mathrm{rf}} ;$ by reducing $\Delta a$ and adjusting the irradiation time the same amount of energy can be imparted to the parent ion. Figure 1a demonstrates the SORI dissociation of the cyclic decapeptide gramicidin $\mathrm{S}$ in which a single activation frequency was applied $1000 \mathrm{~Hz}$ off-resonance relative to the $[\mathrm{M}+\mathrm{H}]^{+}$observed at $m / z 1142$. The resulting fragmentation is typical of SORI activation of proteins and peptides: low energy dissociation products are favored, which results in mostly $\mathrm{Y}$ - and B-type frag-
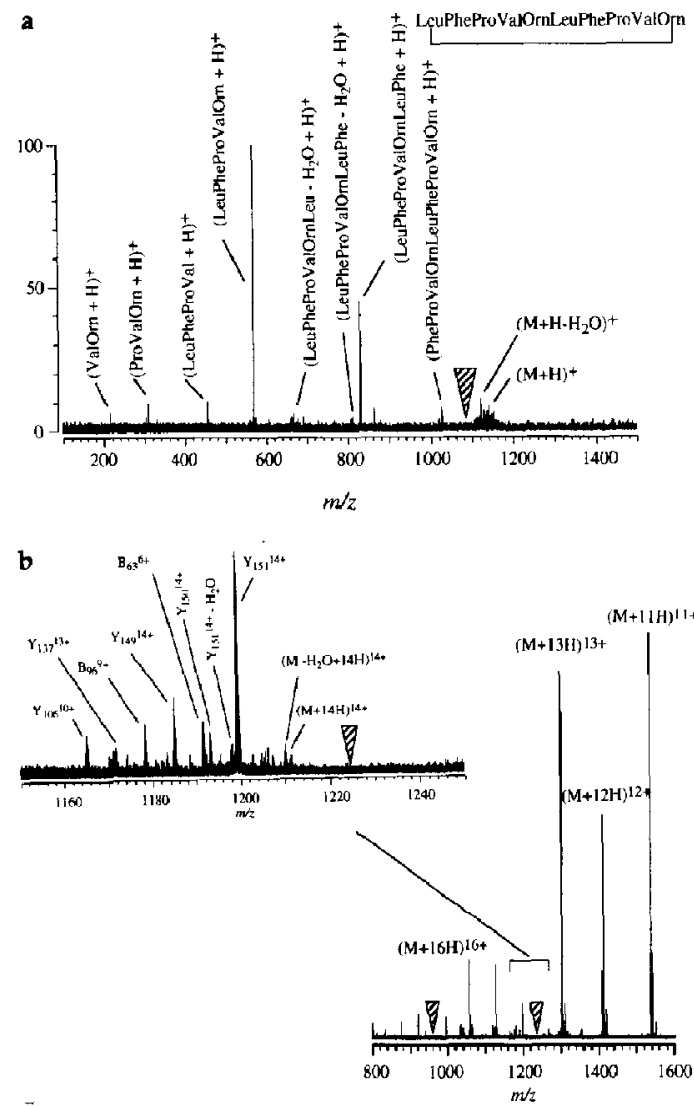

Figure 1. (a) Representative spectrum for sustained offresonance irradiation (SORI) for collision-activated dissociation of singly protonated gramicidin $\mathrm{S}(\mathrm{m} / 21141)$ with $1 \times 10^{-5}$-torr $\mathrm{N}_{2}$ as target and 500-ms, $8.5-\mathrm{V}$ electric field pulse, $1000 \mathrm{~Hz}$ above $(m / z$ 1129.09) the natural cyclotron frequency of the singly charged parent ion. (b) Multiple frequency SORI of the [M + $14 \mathrm{H}]^{14+}$ ion $(m / z$ 1211) of equine apomyoglobin with irradiation pulses at $m / z 945.37$ and 1225.42. Target gas peak presstre, pulse amplitude, and duration are same as in (a). The average resolution of 50,000 (full width at half maximum) is more than adequate to resolve the 1-u spacing of the isotopic peaks and provide unambiguous charge state determination.

ments. Nomenclature used for oligopeptide dissociation products is based on conventional notation [25] modified to indicate charge state [26].

\section{Multiple Frequency Sustained Off-Resonance Irradiation}

This approach takes advantage of the superposition principle, which states that two or more waves can traverse space independently of one another. In the case of SORI activation, multiple activation frequencies can be applied at different frequencies and will act independently of each other. In many applications, independent multiple sustained off-resonance irradiation can have distinct advantages over conventional 
SORI activation. For example, two activation frequencies can be applied in concert, dissociating the parent ion and a selected daughter ion as it is formed, thus providing significantly enhanced sequence information. When a distribution of charge states is present, as is common with electrospray ionization, it may be advantageous to irradiate several charge states simultaneously (and at different $\pm \Delta \omega$ ) to generate increased fragmentation. As demonstrated below, a peptide-protein mixture can be separated on-line and subjected to a customized SORI waveform that contains at least one activation frequency for each component. Figure $1 \mathrm{~b}$ demonstrates the SORI dissociation of the $\left[\mathrm{M}+14 \mathrm{H}^{\dagger}\right]^{14+}$ charge state of equine apomyoglobin irradiated at both $m / z 1225.42(-1000 \mathrm{~Hz}$ offresonance relative to the $\left[\mathrm{M}+14 \mathrm{H}^{+}\right]^{14+}$ charge state of equine apomyoglobin) and $m / z 945.37(-1000 \mathrm{~Hz}$ off-resonance relative to the $\left[\mathrm{M}+3 \mathrm{H}^{+}\right]^{3+}$ charge state of bee venom melittin). The average resolution of the resulting fragment ions is $\approx 50,000$ full width at half maximum, which is more than adequate to resolve the $1-\mathrm{u}$ spacing of the ${ }^{13} \mathrm{C}$ isotope peaks and provide unambiguous charge state determination. No appreciable change in the dissociation of the $\left[\mathrm{M}+14 \mathrm{H}^{+}\right]^{14+}$ charge state is observed in the presence of additional irradiation frequencies provided that the additional frequencies are adequately separated.

The limitations of SORI activation can be magnified in multiple frequency SORI activation because each independent frequency that comprises the waveform creates a new "blind spot" in the resulting mass spectrum. An additional logistical concern of the multiple frequency approach is that when a relatively large number of irradiation frequencies are combined, the initial phase relationship can result in a large initial "beat" in the waveform that is due to constructive interference of the frequencies; in some cases, impulse excitation and subsequent ion loss result. Although phase scrambling or randomization will alleviate this problem, the application of a simple apodization windowing function to the waveform prevents any unnecessary impulse excitation. A simple three frequency SORI waveform is shown in Figure 2 as is the corresponding frequency (mass-to-charge ratio) domain spectrum. The time domain spectrum has been apodized to minimize boundary effects. Because of the irradiation bandwidths and memory limitations of the arbitrary function generator, the waveform was limited to $82 \mathrm{~ms}$. Because SORI and multiple frequency SORI are best carried out over longer time scales (typically 500 to $2000 \mathrm{~ms}$ ), the waveform in Figure 1 was repeated for $500 \mathrm{~ms}$ during activation.

\section{Multiple Frequency SORI in Conjunction with On-Line Separations}

Figure 3 illustrates the potential of multiple frequency SORI for acquiring partial (or potentially complete) sequence information from individual components of a a

b
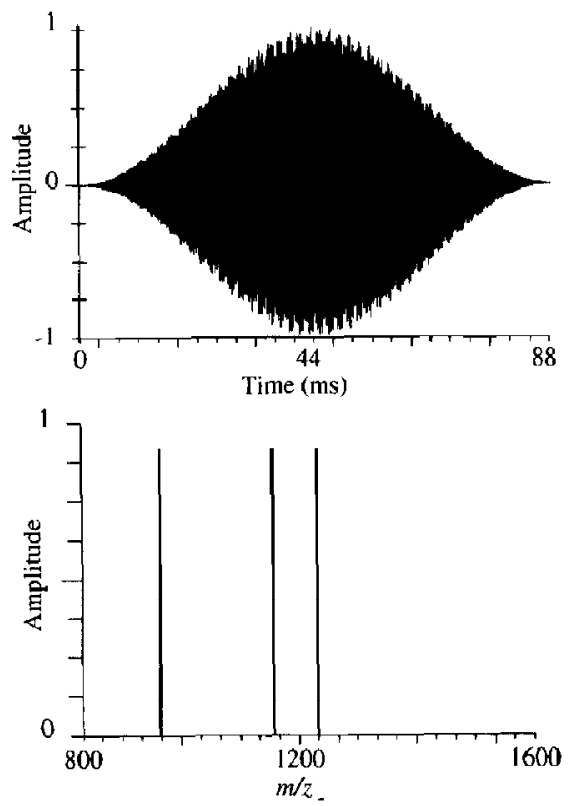

Figure 2. (a) Time domain multiple frequency SORI waveform consisting of three frequencies that correspond to $m / z$ 945.37, 1147.0, and 1225.42. (b) Mass domain spectrum of (a) obtained by Fourier transformation of the time-domain waveform.

complex mixture. The mixture was first analyzed without SORI dissociation to determine the mass-to-charge ratio of the molecular ions for each component. Figure $3 a$ depicts the resulting total ion electropherogram (TIE). A SORI waveform was generated that contained one activation frequency for each of the three major components of the mixture (each irradiation frequency was $\pm 1000 \mathrm{~Hz}$ off-resonance relative to the selected molecular ion). The addition of the multiple frequency SORI activation pulse sequence required only slight modification to the standard CE-FTTCR pulse sequence because the waveform was applied during the high pressure ion accumulation event. The duration of this injection-dissociation event was increased from 50 to $500 \mathrm{~ms}$ to allow sufficient activation.

As shown in Figure 3b, the application of the multiple frequency SORI waveform induces extensive fragmentation of the parent ion and yields a significant amount of sequence information. The blind spot phenomenon is clearly demonstrated by the absence of the $\mathrm{Y}_{16}^{2+}$ fragment ion $(m / z=947.7)$, which lands in close proximity to one of the irradiation frequencies $(\mathrm{m} / z=$ 945.37). Moving the irradiation frequency and concomitant blind spot to $-1000 \mathrm{~Hz}(\mathrm{~m} / z=957.98)$ allows observation of the $Y_{16}^{2+}$ fragment and precludes observation of the $B_{10}^{+}$fragment. In most cases, multiple charge states of the molecular ion are present; thus no molecular weight information is sacrificed. This on-line sequencing approach is similar to that presented by Ramsey et al. [27] in which random noise was applied to the endcaps of a quadrupole ion trap 


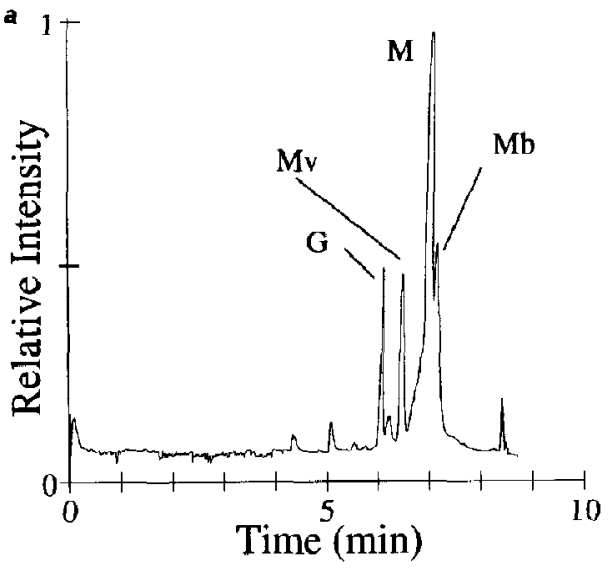

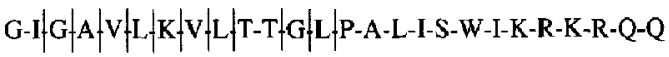
b

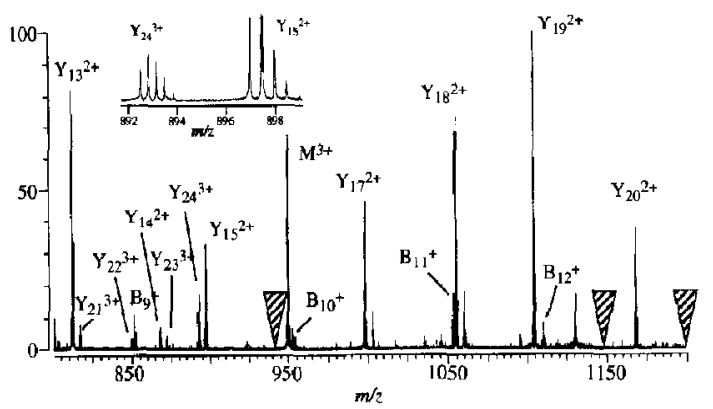

Figure 3. (a) Total ion electropherogram for separation of a mixture that contains gramicidin $S$, melittin, and equine apomyoglobin labeled $G, M$, and $M b$, respectively (see text for $C E$ parameters). The analyte peak appearing at $\sim 6.5$ min corresponds to a melittin variant $M_{v}$.. (b) Multiple frequency SORI dissociation products from component 3 , bee venom melittin ( $2845 \mathrm{MW})$, that yield partial sequence information $(\approx 50 \%)$ with a single dissociation event. The average resolution of 50,000 (full width at half maximum) is more than adequate to resolve the 1-u spacing of the isotopic peaks and provide unambiguous charge state determination.

[28] subsequent to separation by capillary electrophoresis. Similarly, Moseley et al. [29] reported the application of $\mathrm{CE} / \mathrm{ESI}$ tandem mass spectrometry analysis to obtain sequence information for a mixture of neuropeptides via a triple sector quadrupole mass spectrometer. Dissociation by multiple frequency SORI activation has the unique advantage of specificity, compared to random noise activation or capillaryskimmer dissociation, because only species within a well defined mass-to-charge ratio range are activated. Thus, in situations where two closely related components are not amenable to efficient separation by $\mathrm{CE}$, the co-eluting species can be dissociated independently by applying different IMSORI waveforms during replicate runs. For example, consider the case of two peptides that differ by a single amino acid substitution in which the substitution involves two similar amino acids (e.g., Gly for Ala). The nearly identical electrophoretic mobilities of the peptides makes efficient highly resolved separation problematic. Complete or partial sequence information still can be acquired by performing replicate experiments in which the low mass-to-charge ratio species is irradiated at $+\Delta \omega$ (lower mass-to-charge ratio) followed by the irradiation of the high mass-to-charge ratio species at $-\Delta \omega$ (higher mass-to-charge ratio).

Although one multiple frequency SORI dissociation event most likely will not yield complete sequence information for larger peptides, the ability to perform nondestructive ion remeasurement and $\mathrm{MS} / \mathrm{MS}^{n}$ can lead to enhanced sequence information. Although acquisition of complete sequence information is preferred, determination of the sequence of a contiguous run of 5 to 10 amino acids is often adequate to recognize a unique DNA motif in the genome of a living organism [30]. Thus, specific sequence information could be used to design amplification primers (i.e., DNA probes) for the polymerase chain reaction [31]. Consequently, this allows the characterization of mutations, polymorphisms, and evolutionary changes in the DNA sequence [32]. In addition, direct sequencing can be carried out by application of the triple primer method, where the first two flanking primers amplify the DNA segment enzymatically and then the third primer (labeled with ${ }^{32} \mathrm{P}$ ) is used to sequence the amplified DNA fragment directly [32b]. We are presently investigating the feasibility of computer-mediated multiple frequency SORI dissociation of chromatographic and electrophoretic effluents in which dissociation frequencies are chosen in real time relative to the separation. This enhanced functionality, applied in conjunction with stored waveform inverse Fourier transform isolation of fragment ions, should provide a viable method for performing MS/MS ${ }^{n}$ on very small amounts of sample from a complex mixture without sacrificing mass spectrometric or chromatographic performance.

\section{Acknowledgments}

The authors thank Mike Senko and Professor Fred McLafferty for an enlightening discussion on SORI techniques and for sharing initial ESI-SORI data. Additional input from Dr. A. L. Rockwood, Dr. M. G. Sherman, Dr. H. R. Udseth, and Dr. S. Van Orden is greatly appreciated. This research was supported by internal PNL exploratory research through the U.S. Department of Energy. Pacific Northwest Laboratory is operated by Battelle Memorial Institute for the U.S. Department of Energy under contract DE-AC06-76RLO 1830.

\section{References}

1. Henry, K. D.; Williams, E. R.; Wang, B. H.; McLafferty, F. W.; Shabanowitz, J.; Hunt, D. F. Proc. Natl. Acad. Sci. USA 1989, $86,9075-9078$.

2. Henry, K. D.; McLafferty, F. W. Org. Mass Spectrom. 1990, 25 , 490-492.

3. Hofstadler, S. A.; Wahl, J. H.; Bruce, J. E.; Smith, R. D. J. Am. Chem. Soc. 1993, 115, 6983-6984. 
4. Winger, B. E; Hofstadler, S. A; Bruce, J. E.; Udseth, H. R.; Smith, R. D. J. Am. Soc. Mass Spectrom. 1993, 4, 566-577.

5. Bruce, J. E.; Anderson, G. A.; Hofstadler, S. A.; Winger, B. E.; Smith, R. D. Rapid Commun. Mass Spectrom. 1993, 7, 700-703

6. Beu, S. C.; Senko, M. W.; Quinn, J. P.; McLafferty, F. W. J. Am. Soc. Mass Spectrom. 1993, 4, 190-192.

7. Beu, S. C.; Senko, M. W.; Quinn, J. P.; Wampler, F. M.; McLafferty, F. W. J. Am. Soc. Mass Spectrom. 1993, 4, 557-565

8. Loo, J. A.; Quinn, J. P.; Ryu, 5. 1.; Henry, K. D.; Senko, M. W.; McLafferty, F. W. Proc. Natl. Acad. Sci. USA 1992, 89, 286-289

9. McLafferty, F. W.; Beu, S. C.; Senko, M. W.; O'Connor, P. B. Proceedings of the 41st ASMS Conference on Mass Spectrometry and Alied Topics; San Francisco, CA, 1993; pp 797a-797b.

10. Bruce, J. E; Hofstadler, S. A.; Winger, B. E.; Smith, R. D. Int. J. Mass Spectrom. Ion Processes 1994, 132, 97-107.

11. Guan, Z. Q.; Hofstadler, S. A.; Laude, D. A. Anul. Cherr. 1993, $65,1588-1593$.

12. Ijames, C. F.; Wilkins, C. L. Anal. Chem. 1990, 62, 1295-1299; Castoro, J. A.; Rucker, P. V.; Wilkins, C. L. J. Am. Soc. Mass Spectrom. 1992, 3, 445-450; Cooks, R. G.; Ast, T.; Mabud, M. A. Int. t. Mass Spectrum. Ion Processes 1990, 100, 209-265.

13. McCormack, A. L.; Jones, J. L.; Wysocki, V. H. J. Am. Soc. Mass Spectrom. 1992, 3, 859-862; Schey, K. L.; Thornburg, K. R. Proceedings of 41 st ASMS Conference om Mass Spectrometry and Allied Topics; San Francisco, CA, 1993; pp 269a-269b.

14. Gauthier, J. W.; Trautman, T, R.; Jacobson, D. B. Anal. Chim. Acta 1991, 246, 211-225.

15. Bruin, G. L. Mir Huiden, R.; Kraak, J. C.; Poppe, H. I, Chrom. 1989, 480, 339-343.

16. Lukacs, K. D. Diss. Abstr. Int. 1983, 44, 3766.

17. Wahl, J. H.; Gale, D. C.; Smith, R. D. J. Chromatogr. 1994, 659, 217-222.

18. Wahl, J. H.; Gale, D. C.; Hofstadler, S. A.; Udseth, H. R.; Smith, R. D. Proceedings of 41st ASMS Conference on Mass Spectrometry and Allied Topics; San Francisco, CA, 1993; pp $1045 \mathbf{a}-1045 \mathbf{b}$.
19. McLafferty, F. W., Ed. Tandem Mass Spectrometry; Wiley: New York, 1983; Busch, K. L.; Glish, G. L.; McLuckey, S. A. Mass Spectrometry / Mass Spectrometry; VCH: New York, 1988.

20. Busman, M.; Rockwood, A. L.; Smith, R. D. J. Phys. Chem. 1992, 96, 2397-2400; Smith, R. D.; Barinaga, C. J.; Udseth, H. R. I. Phys. Chem. 1989, 93, 5019-5022; Griffin, L. L.; McAdoo, D. J. J. Am. Soc. Mass Spectrom. 1993, 4, 11-15.

21. Baykut, G; Watson, C. H.; Weller, R. R.; Eyler, J. R. J. Am. Chem. Soc. 1985, 107, 8036-8039.

22. Burnier, R. C.; Cody, R. B.; Freiser, B. S. J. Am. Chem. Soc, 1982, 104, 7436-74.39.

23. Boering, K. A.; Rolfe, J.; Brauman, J. I. Ropid Commun. Mass Spectrom. 1992, 6, 303-305.

24. Lee, S. A.; Jian, C. Q.; Huang, Y. Q.; Freiser, B. S. Rapid Commun. Mass Spectrom. 1993, 7, 819-821.

25. Roepstorff, P.; Fohlman, J. Biomed. Mass Spectrom. 1984, 11 . 601; Biemann, K. Biomed. Mass Spectrom. 1988, 16, 99-111.

26. Barinaga, C. J.; Edmonds, C. G.; Udseth, H. R.; Smith, R. D. Rapid Commun. Mass Spectrom. 1989, 3, 160-164

27. Ramsey, R. S.; Asano, K. G.; Hart, K. J.; Goeringer, D. E.; Van Berkel, G. J.; McLuckey, 5. A. Proceedings of 41st ASMS Conference on Mass Spectrometry and Allied Topics; San Francisco, CA, 1993; pp 749a-749b.

28. Brown, R. S.; Gilfrich, N. L. Appl. Spectrosc. 1993, 47, 103-110.

29. Moseley, M. A.; Jorgenson, J. W.; Shabanowitz, J.; Hunt, D. F.; Tomer, K. B. I. Am. Soc. Mass Spectrom. 1992, 3, 289-300.

30. Nguyen, T. T.; Helene, C. Angew. Chem. Int. Ed. Engl. 1993, 32, 666-690.

31. Saiki, R. K.; Scharf, S.; Faloona, F.; Mullis, K. B.; Horn, G. T.; Erlich, H. A.; Arnheim, N. Science 1985, 230, 1350-1354; Mullis, K. B. Sci. Am. 1990, April, 56-65; Erlich, H. A.; Gelfand, D.; Sninsky, J. J. Science 1991, 252, 1643-1651 and references cited therein.

32. (a) Wong, C.; Dowling, C. E.; Saiki, R. K.; Higuchi, R. G.; Erlich, H. A.; Kazazian, H. H. Nature 1987, 330, 384-386; (b) Wrischnik, L. A.; Higuchi, R. G.; Stoneking, M.; Erlich, H. A.; Arnheim, N.; Wilson, A. C. Nucleic Acids Res. 1987, 15, $529-542$. 